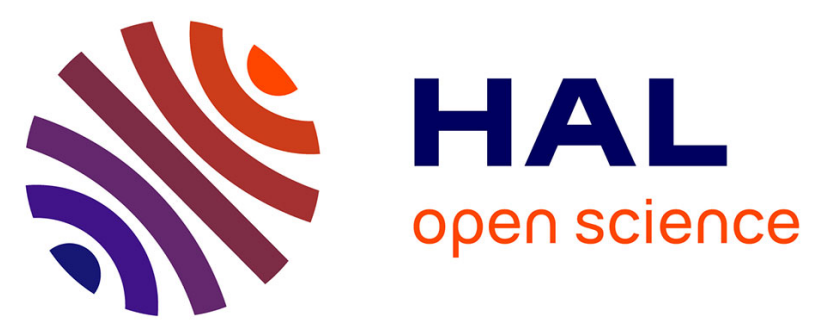

\title{
Relations entre l'état angulaire d'une vapeur atomique soumise au pompage optique et ses propriétés d'absorption et de dispersion - Seconde Partie
} F. Laloë, M. Leduc, P. Minguzzi

\section{- To cite this version:}

F. Laloë, M. Leduc, P. Minguzzi. Relations entre l'état angulaire d'une vapeur atomique soumise au pompage optique et ses propriétés d'absorption et de dispersion - Seconde Partie. Journal de Physique, 1969, 30 (4), pp.341-349. 10.1051/jphys:01969003004034100 . jpa-00206792

HAL Id: jpa-00206792

https://hal.science/jpa-00206792

Submitted on 1 Jan 1969

HAL is a multi-disciplinary open access archive for the deposit and dissemination of scientific research documents, whether they are published or not. The documents may come from teaching and research institutions in France or abroad, or from public or private research centers.
L'archive ouverte pluridisciplinaire HAL, est destinée au dépôt et à la diffusion de documents scientifiques de niveau recherche, publiés ou non, émanant des établissements d'enseignement et de recherche français ou étrangers, des laboratoires publics ou privés. 


\title{
RELATIONS ENTRE L'ÉTAT ANGULAIRE D'UNE VAPEUR ATOMIQUE SOUMISE AU POMPAGE OPTIQUE ET SES PROPRIÉTÉS D'ABSORPTION ET DE DISPERSION
}

\author{
Seconde Partie (1) \\ Par F. LALÖ̈, M. LEDUG et P. MINGUZZI $\left({ }^{2}\right)$, \\ Faculté des Sciences de Paris, Laboratoire de Spectroscopie Hertzienne de l'E.N.S., associé au C.N.R.S.
}

(Reçu le 31 octobre 1968.)

\begin{abstract}
Résumé. - On étudie dans cet article le lien qui existe entre l'anisotropie des propriétés optiques d'une vapeur atomique et les propriétés angulaires des atomes dans l'état fondamental. La direction du faisceau lumineux qui se propage dans la vapeur, ainsi que l'orientation et l'alignement des atomes sont quelconques; l'influence de chaque observable de l'état fondamental est discutée de manière détaillée à partir d'un modèle géométrique. Ce dernier fait intervenir d'une manière qui est précisée au cours de l'article un vecteur pour caractériser l'orientation, un ellipsoïde pour l'alignement. On retrouve ainsi un certain nombre de prévisions théoriques établies de manière différente dans un précédent article.

Absiract. - In this paper, we study the relation between the anisotropic optical properties of an atomic vapour and the angular characteristics of the atomic ground state. The direction of the light beam travelling through the vapour, as well as the orientation and alignment of the atoms are set at no particular value. The influence of every ground state observable is discussed in detail within the frame of a geometrical model. It involves a vector and an ellipsoid to describe respectively the atomic orientation and alignment. One establishes in this way a number of results which are identical to those obtained previously from a different view-point.
\end{abstract}

\section{INTERPRETATION GEOMETRIQUE}

Dans la première partie de cette étude [1], l'influence sur le signal optique des diverses grandeurs atomiques dans l'état fondamental (orientation, alignement) a été étudiée à partir d'un calcul qui fait intervenir le formalisme des opérateurs tensoriels irréductibles. Cependant, dans un certain nombre de publications antérieures [2], [3], [4], l'interprétation de certains signaux de détection optique est faite de manière toute différente : elle utilise une représentation géométrique de la polarisation des atomes dans l'état fondamental; cette polarisation est notamment caractérisée par un vecteur, qui définit l'orientation des atomes et qui se comporte dans un champ $\mathbf{H}_{0}$ comme un dipôle magnétique. Or, dans le cas général, l'état angulaire de la vapeur ne peut être caractérisé par la seule donnée des trois composantes d'un vecteur; on voit donc que les représentations géométriques qui viennent d'être citées ne sont pas toujours suffisantes (elles le

(1) La première partie de cette étude a paru dans le précédent numéro du Journal de Physique.

(2) Istituto di Fisica dell'Università, Pisa, Gruppo Nazionale di Struttura della Materia del C.N.R. sont lorsque l'état fondamental ne comprend que deux sous-niveaux; c'est par exemple le cas de ${ }^{199} \mathrm{Hg}$ ). C'est pourquoi nous allons montrer, dans la deuxième partie de ce travail, comment on peut donner une interprétation géométrique utilisable dans le cas général des propriétés optiques d'une vapeur. Nous verrons qu'aux trois paramètres qui définissent l'orientation on peut associer un vecteur, alors que les cinq paramètres dont dépend l'alignement conduisent à introduire un ellipsoïde.

Les équations qui sont données dans la première partie ne permettent pas d'introduire aisément de telles considérations géométriques. C'est pourquoi nous consacrons le paragraphe suivant à mettre les résultats algébriques déjà obtenus sous une forme légèrement différente, dans laquelle la direction du faisceau lumineux détecteur ne joue plus un rôle privilégié.

A. Introduction de la matrice $M$. - Introduisons un système d'axes Oxyz, indépendants du faisceau lumineux, et liés par exemple au champ magnétique statique. L'état angulaire de la vapeur atomique est alors défini par les valeurs moyennes dans l'état fondamental d'un certain nombre d'opérateurs tensoriels 
$U_{q}^{(k)}$ dont les composantes standards relatives aux axes Oxyz ont pour expression :

$$
U_{q}^{(k)}=\sum_{q^{\prime}+q^{\prime \prime}=q}\left\langle 11 q^{\prime} q^{\prime \prime} \mid k q\right\rangle D_{q^{\prime}} P_{e} D_{q^{\prime \prime}}
$$

Les opérateurs $U_{a}^{(k)}$ s'obtiennent par rotation à partir des opérateurs $T_{q}^{(k)}$ définis en (I.A.11).

La matrice $B(t)$ utilisée dans la première partie agit dans le sous-espace $\mathscr{F}_{\mathrm{XY}}^{(2)}$ des vecteurs polarisation contenus dans le plan XOY. Par généralisation de $B(t)$, on introduit un opérateur $M$, agissant dans l'espace à trois dimensions de toutes les polarisations lumineuses possibles, dont les éléments de matrice sont définis par :

$$
\left\langle\mathbf{e}_{i}|M| \mathbf{e}_{j}\right\rangle=\operatorname{Tr}_{\mathrm{f}}\left\{\sigma_{\mathrm{f}}(t)\left(\mathbf{e}_{i}^{*} \cdot \mathbf{D}\right) P_{e}\left(\mathbf{e}_{j} \cdot \mathbf{D}\right)\right\}
$$

$M$ est une matrice hermitique, définie positive.

Considérons une direction quelconque $\mathrm{OZ}$ de propagation du faisceau lumineux détecteur, à laquelle correspond l'opérateur $P_{X Y}$ de projection sur les vecteurs polarisation du plan XOY. $B$ et $M$ sont reliés par l'égalité :

$$
B=P_{X Y} M P_{X Y}
$$

$B$ est la restriction de $M$ au sous-espace $\mathscr{F}_{X Y}^{(2)}$. En utilisant (I.A.1) et (II.A.3), on obtient :

$$
\begin{aligned}
\pi_{\mathrm{T}}-\pi_{\mathrm{I}}=-\alpha P_{X Y} & \left\{\frac{\Gamma^{\prime}}{2}\left[M, \pi_{\mathrm{I}}\right]_{+}\right. \\
& \left.+i \Delta E^{\prime}\left[M, \pi_{\mathrm{I}}\right]\right\} P_{X \mathrm{Y}}
\end{aligned}
$$

Par comparaison avec (I.A.1), on voit que $M$ joue ici un rôle analogue à celui de $B$ dans la première partie.

Il est possible de calculer les éléments de la matrice $M$ en fonction des valeurs moyennes :

$$
\left\langle U_{q}^{(k)}\right\rangle=\operatorname{Tr}_{\mathrm{f}}\left\{\sigma_{\mathrm{f}} U_{q}^{(k)}\right\}
$$

des opérateurs $U_{q}^{(k)}$ définis plus haut. Soient $\left|\mathbf{e}_{x}\right\rangle$, $\left|\mathbf{e}_{y}\right\rangle$ et $\left|\mathbf{e}_{z}\right\rangle$ les trois vecteurs de $\mathscr{F}^{(3)}$ correspondant aux polarisations planes parallèles à $\mathrm{Ox}$, $\mathrm{Oy}$ et $\mathrm{Oz}$; on pose :

$$
\left\{\begin{array}{l}
\left|\mathbf{e}_{+}\right\rangle=-\frac{1}{\sqrt{2}}\left(\left|\mathbf{e}_{x}\right\rangle+i\left|\mathbf{e}_{y}\right\rangle\right) \\
\left|\mathbf{e}_{0}\right\rangle=\left|\mathbf{e}_{z}\right\rangle \\
\left|\mathbf{e}_{-}\right\rangle=\frac{1}{\sqrt{2}}\left(\left|\mathbf{e}_{x}\right\rangle-i\left|\mathbf{e}_{y}\right\rangle\right) .
\end{array}\right.
$$

$\left|\mathbf{e}_{+}\right\rangle,\left|\mathbf{e}_{0}\right\rangle$ et $\left|\mathbf{e}_{-}\right\rangle$correspondent, dans les axes Oxyz, aux polarisations $\sigma_{+}, \pi$ et $\sigma_{-}$. On peut décomposer $M$ en trois parties en posant :

$$
M=M^{(0)}+M^{(1)}+M^{(2)}
$$

où les trois matrices hermitiques $M^{(0)}, M^{(1)}$ et $M^{(2)}$ ont pour expression, dans la base $\left|\mathbf{e}_{+}\right\rangle,\left|\mathbf{e}_{0}\right\rangle,\left|\mathbf{e}_{-}\right\rangle$:

$$
\begin{aligned}
& M^{(0)}=-\frac{1}{\sqrt{3}}\left\langle U_{0}^{(0)}\right\rangle \times 1 \quad \text { (1 est la matrice unité à trois dimensions) } \\
& M^{(1)}=\frac{1}{\sqrt{2}}\left(\begin{array}{ccc}
\left\langle U_{0}^{(1)}\right\rangle & \left\langle U_{-1}^{(1)}\right\rangle & 0 \\
-\left\langle U_{1}^{(1)}\right\rangle & 0 & \left\langle U_{-1}^{(1)}\right\rangle \\
0 & -\left\langle U_{1}^{(1)}\right\rangle & -\left\langle U_{0}^{(1)}\right\rangle
\end{array}\right) \\
& M^{(2)}=\left(\begin{array}{ccc}
-\frac{1}{\sqrt{6}}\left\langle U_{0}^{(2)}\right\rangle & -\frac{1}{\sqrt{2}}\left\langle U_{-1}^{(2)}\right\rangle & -\left\langle U_{-2}^{(2)}\right\rangle \\
\frac{1}{\sqrt{2}}\left\langle U_{1}^{(2)}\right\rangle & \sqrt{\frac{2}{3}}\left\langle U_{0}^{(2)}\right\rangle & \frac{1}{\sqrt{2}}\left\langle U_{-1}^{(2)}\right\rangle \\
-\left\langle U_{2}^{(2)}\right\rangle & -\frac{1}{\sqrt{2}}\left\langle U_{1}^{(2)}\right\rangle & -\frac{1}{\sqrt{6}}\left\langle U_{0}^{(2)}\right\rangle
\end{array}\right)
\end{aligned}
$$

$M^{(0)}$ ne dépend que de la population totale de l'état fondamental, $M^{(1)}$ que de son orientation et $M^{(2)}$ que de son alignement. Nous allons étudier successivement l'influence de ces trois matrices sur la modification de la matrice polarisation lumineuse.

B. Influence de la population totale de l'état fondamental. $-M^{(0)}$ est définie par l'équation (II.A.7), où $\left\langle U_{0}^{(0)}\right\rangle$ est une constante, égale à $c^{(0)}$. Si l'on pose :

$$
\begin{aligned}
\left\langle D^{2}\right\rangle=\operatorname{Tr}_{\mathrm{f}}\left\{\sigma_{\mathrm{f}}(t)\right. & {\left[D_{x} P_{e} D_{x}\right.} \\
& \left.\left.+D_{y} P_{e} D_{y}+D_{z} P_{e} D_{z}\right]\right\}
\end{aligned}
$$

on obtient :

$$
M^{(0)}=\frac{1}{3}\left\langle D^{2}\right\rangle \times 1
$$


$M^{(0)}$ est donc une matrice scalaire, constante et positive. On voit alors sur (II.A.4) que $M^{(0)}$ correspond à une absorption isotrope de la lumière, indépendante de la direction de propagation du faisceau lumineux, de sa polarisation et de l'état angulaire de la vapeur.

C. Influence de l'orientation. - L'expression de la matrice $M^{(1)}$ est donnée en (II.A.8), dans la base $\left|\mathbf{e}_{+}\right\rangle,\left|\mathbf{e}_{0}\right\rangle,\left|\mathbf{e}_{-}\right\rangle$; il est commode d'écrire $M^{(1)}$ dans la base $\left|\mathbf{e}_{x}\right\rangle,\left|\mathbf{e}_{y}\right\rangle,\left|\mathbf{e}_{z}\right\rangle$, en fonction des composantes du moment cinétique $\langle\vec{I}\rangle$ dans l'état fondamental. On obtient, en utilisant l'égalité (I.A.12) :

$M^{(1)}$

$=i \frac{c^{(\mathbf{1})}}{\sqrt{2}}\left(\begin{array}{ccc}0 & -\left\langle I_{z}\right\rangle & \left\langle I_{y}\right\rangle \\ \left\langle I_{z}\right\rangle & 0 & -\left\langle I_{x}\right\rangle \\ -\left\langle I_{y}\right\rangle & \left\langle I_{x}\right\rangle & 0\end{array}\right)$.

On voit que l'action de l'opérateur $M^{(1)}$ sur un vecteur polarisation $\left|\mathbf{e}_{\lambda_{0}}\right\rangle$ quelconque s'écrit :

$$
M^{(\mathbf{1})}\left|\mathbf{e}_{\lambda_{0}}\right\rangle=i \frac{c^{(\mathbf{1})}}{\sqrt{2}}|\mathbf{I}\rangle \wedge\left|\mathbf{e}_{\lambda_{0}}\right\rangle
$$

où $|\mathbf{I}\rangle$ est le vecteur de $\mathscr{F}^{(3)}$ défini par :

$$
\begin{aligned}
|\mathbf{I}\rangle=\left\langle I_{x}\right\rangle\left|\mathbf{e}_{x}\right\rangle+\left\langle I_{y}\right\rangle\left|\mathbf{e}_{y}\right\rangle+\left\langle I_{z}\right\rangle\left|\mathbf{e}_{z}\right\rangle \\
=\left\langle I_{X}\right\rangle\left|\mathbf{e}_{X}\right\rangle+\left\langle I_{Y}\right\rangle\left|\mathbf{e}_{Y}\right\rangle \\
\quad+\left\langle I_{Z}\right\rangle\left|\mathbf{e}_{Z}\right\rangle . \quad \text { (II }
\end{aligned}
$$

La formule (II.C.2) permet de calculer aisément l'action sur $\left|\mathbf{e}_{\lambda_{0}}\right\rangle$ de $B^{(1)}=P_{X Y} M^{(1)} P_{X Y}$ :

$B^{(1)}\left|\mathbf{e}_{\lambda_{0}}\right\rangle=i \frac{c^{(1)}}{\sqrt{2}}\left\langle I_{Z}\right\rangle\left|\mathbf{e}_{Z}\right\rangle \wedge\left|\mathbf{e}_{\lambda_{0}}\right\rangle$.

Pour interpréter cette égalité, il est utile de rappeler la signification physique du vecteur $B\left|\mathbf{e}_{\lambda_{0}}\right\rangle$, dans le cas des épaisseurs optiques faibles : on montre dans JP3 et JP4 que la modification de la matrice polarisation du faisceau lumineux, lors de la traversée de la vapeur atomique, peut être obtenue en ajoutant au champ électrique de l'onde incidente le champ rayonné en avant par les atomes, ce qui revient à ajouter au vecteur polarisation $\left|\mathbf{e}_{\lambda_{0}}\right\rangle$ un vecteur $\left|\mathbf{e}^{R}\right\rangle$, très petit devant $\left|\mathbf{e}_{\lambda_{0}}\right\rangle$, et donné par :

$$
\left|\mathbf{e}^{R}\right\rangle=-\alpha\left(\frac{\Gamma^{\prime}}{2}+i \Delta E^{\prime}\right) B\left|\mathbf{e}_{\lambda_{0}}\right\rangle \text {. }
$$

(II.C.4) nous permet donc de calculer la contribution de l'orientation au champ électrique rayonné par la vapeur, qui correspond à :

$$
\begin{aligned}
\left|\mathbf{e}^{R,(1)}\right\rangle= & -\alpha\left(\frac{\Gamma^{\prime}}{2}+i \Delta E^{\prime}\right) B^{(1)}\left|\mathbf{e}_{\lambda_{0}}\right\rangle \\
= & \frac{\alpha c^{(1)}}{\sqrt{2}}\left(\Delta E^{\prime}-i \frac{\Gamma^{\prime}}{2}\right) \\
& \left\langle I_{Z}\right\rangle\left|\mathbf{e}_{Z}\right\rangle \wedge\left|\mathbf{e}_{\lambda_{0}}\right\rangle .
\end{aligned}
$$

Nous retrouvons donc un résultat, établi dans JP4 [5], que nous avons utilisé dans la première partie : les composantes de l'orientation dans le plan XOY ne contribuent pas au signal optique. De plus, on voit sur (II.C.5) que l'effet de $B^{(\mathbf{1})}$ sur le vecteur $\left|\mathbf{e}_{\lambda_{0}}\right\rangle$ correspond à une rotation infinitésimale autour de $\mathrm{OZ}$; les polarisations principales de la vapeur orientée sont donc les deux polarisations circulaires droite et gauche par rapport à $\mathrm{OZ}$ : nous retrouvons le lien entre l'orientation $\left\langle I_{Z}\right\rangle$ et les propriétés de dichroïsme et de biréfringence circulaires de la vapeur.

Enfin, lorsque $\left|\mathbf{e}_{\lambda_{0}}\right\rangle$ correspond à une polarisation linéaire, nous pouvons retrouver géométriquement un certain nombre de résultats de la partie I. L'expression (II.C.5) montre qu'alors le champ rayonné par la vapeur a une polarisation linéaire, perpendiculaire à la polarisation du faisceau incident (nous retrouvons ainsi un résultat établi par Happer et Mathur [6]).

$\mathrm{Si}$ le faisceau lumineux détecteur est résonnant, le champ rayonné est en quadrature de phase avec le champ incident : la polarisation transmise est donc légèrement elliptique, mais la projection du champ total sur $\left|\mathbf{e}_{\lambda_{0}}\right\rangle$ ne varie pas au premier ordre : la quantité de lumière absorbée par la vapeur ne dépend donc pas de $\left\langle I_{Z}\right\rangle$. Pour détecter cette observable, il serait nécessaire d'utiliser un analyseur pour mesurer le degré de polarisation circulaire du faisceau transmis.

Si le faisceau détecteur est non résonnant, le champ rayonné est en phase avec le champ incident. Nous voyons donc qu'au premier ordre l'effet de la vapeur sur la lumière se traduit par une rotation du plan de polarisation (effet Faraday).

D. Influence de l'alignement. - 1. MAtrice $M^{(2)}$. - L'expression de la matrice $M^{(2)}$ dans la base $\left|\mathbf{e}_{+}\right\rangle$, $\left|\mathbf{e}_{0}\right\rangle,\left|\mathbf{e}_{-}\right\rangle$est donnée en (II.A.9). Comme dans le cas de l'orientation, calculons cette matrice dans la base $\left|\mathbf{e}_{x}\right\rangle,\left|\mathbf{e}_{y}\right\rangle,\left|\mathbf{e}_{z}\right\rangle$; nous obtenons :
$M^{(2)}=c^{(2)}\left(\begin{array}{c}\left\langle I_{x}^{2}\right\rangle-(1 / 3) I(I+1) \\ (1 / 2)\left\langle I_{x} I_{y}+I_{y} I_{x}\right\rangle \\ \left.(1 / 2)<I_{x} I_{z}+I_{z} I_{x}\right\rangle\end{array}\right.$

$$
\begin{gathered}
(1 / 2)\left\langle I_{x} I_{y}+I_{y} I_{x}\right\rangle \\
\left\langle I_{y}^{2}\right\rangle-(1 / 3) I(I+1) \\
(1 / 2)\left\langle I_{y} I_{z}+I_{z} I_{y}\right\rangle
\end{gathered}
$$

$$
\left.\begin{array}{c}
(1 / 2)\left\langle I_{x} I_{z}+I_{z} I_{x}\right\rangle \\
(1 / 2)\left\langle I_{y} I_{z}+I_{z} I_{y}\right\rangle \\
\left\langle I_{z}^{2}\right\rangle-(1 / 3) I(I+1)
\end{array}\right)
$$


ou encore :

$$
M^{(2)}=c^{(2)}\left[\mathscr{M}^{(2)}-\frac{1}{3} I(I+1)\right]
$$

avec :

$$
\begin{aligned}
& \left\langle\mathbf{e}_{x_{i}}\left|\mathscr{M}^{(2)}\right| \mathbf{e}_{x_{j}}\right\rangle=\frac{1}{2}\left\langle I_{x_{i}} I_{x_{j}}+I_{x_{j}} I_{x_{i}}\right\rangle \\
& \left(x_{i}, x_{j}=x, y, z\right) .
\end{aligned}
$$

Dans cette base, $M^{(2)}$ et $\mathscr{M}^{(2)}$ sont des matrices symétriques réelles : leurs valeurs propres et leurs vecteurs propres sont donc réels, et les polarisations propres correspondant à l'alignement sont linéaires.

Dans le paragraphe précédent, nous avons représenté géométriquement l'action de l'orientation sur les polarisations lumineuses en introduisant un vecteur $|\mathbf{I}\rangle$. Nous allons voir qu'il est commode de faire intervenir dans le cas de l'alignement une représentation géométrique utilisant un ellipsoïde.

2. Ellipsö̈de des Alignements. - a) Définition. Considérons comme donnée la matrice densité des atomes dans l'état fondamental. Nous cherchons à connaître l'alignement $3\left\langle I_{\Omega}^{2}\right\rangle-I(I+1)$ des atomes dans une direction $\Omega$ quelconque. Dans ce but, nous introduisons un point $P$, de coordonnées $x, y, z$, tel que $\overrightarrow{\mathrm{OP}}$ soit parallèle à la direction $\Omega$, et défini par :

$$
|\overrightarrow{\mathrm{OP}}|=r=\frac{1}{\sqrt{\left\langle I_{\Omega}^{2}\right\rangle}}
$$

On a :

$$
I_{\Omega}=\frac{1}{r}\left[x I_{x}+y I_{y}+z I_{z}\right]
$$

et $\left\langle I_{\Omega}^{2}\right\rangle$ est donc donné par :

$$
\left\langle I_{\Omega}^{2}\right\rangle=\sum_{i, j} \frac{x_{i} x_{j}}{2 r^{2}}\left\langle I_{x_{i}} I_{x_{j}}+I_{x_{j}} I_{x_{i}}\right\rangle \quad\left(x_{i}, x_{j}=x, y, z\right) \text {. }
$$

En portant $\left\langle I_{\Omega}^{2}\right\rangle=1 / r^{2}$ dans (II.D.6), on obtient :

$$
\sum_{i, j} \frac{x_{i} x_{j}}{2}\left\langle I_{x_{i}} I_{x_{j}}+I_{x_{j}} I_{x_{i}}\right\rangle=1
$$

qui est l'équation d'un ellipsoïde de centre $O$. On peut encore l'écrire :

$$
(x, y, z) \mathscr{M}^{(2)}\left(\begin{array}{l}
x \\
y \\
z
\end{array}\right)=1
$$

où $\mathscr{M}^{(2)}$ est la matrice définie en (II.D.3).

Le point $\mathrm{P}$ est donc astreint à se déplacer sur un ellipsoïde $\mathscr{E}_{\text {al }}$, défini par une forme quadratique dont la matrice est $\mathscr{M}^{(2)}$ (on peut remarquer l'analogie étroite entre l'ellipsoïde des alignements et l'ellipsoïde d'inertie d'un corps solide, introduit en mécanique rationnelle). La direction du grand axe est celle qui rend $\left\langle I_{\Omega}^{2}\right\rangle$ minimum, celle du petit axe $\left\langle I_{\Omega}^{2}\right\rangle$ maximum. On peut calculer à partir de (II.D.5) la valeur moyenne de $\left\langle I_{\Omega}^{2}\right\rangle$ dans toutes les directions de l'espace; on obtient :

$$
\begin{aligned}
\frac{1}{4 \pi} \int_{4 \pi}\left\langle I_{\Omega}^{2}\right\rangle \mathrm{d} \Omega=\frac{1}{3}\left[\left\langle I_{x}^{2}\right\rangle\right. & \left.+\left\langle I_{y}^{2}\right\rangle+\left\langle I_{z}^{2}\right\rangle\right] \\
& =\frac{1}{3} I(I+1)
\end{aligned}
$$

L'ellipsoïde $\mathscr{E}_{\text {al }}$ est donc en partie intérieur, en partie extérieur à la sphère de centre $\mathrm{O}$ et de rayon $\sqrt{3 / I(I+1)}$.

Notons que, dans le cas général, l'ellipsoïde n'est pas de révolution, de sorte qu'il n'existe pas d'axe autour duquel l'alignement soit invariant par rotation (contrairement à l'orientation). Dans le système des axes principaux de l'ellipsoïde, on a :

$$
\begin{aligned}
\left\langle I_{x} I_{y}+I_{y} I_{x}\right\rangle & =\left\langle I_{y} I_{z}+I_{z} I_{y}\right\rangle \\
& =\left\langle I_{z} I_{x}+I_{x} I_{z}\right\rangle=0 .
\end{aligned}
$$

On montre que ces conditions sont équivalentes à :

$$
\left\{\begin{array}{l}
\left\langle U_{2}^{(2)}\right\rangle=\left\langle U_{-2}^{(2)}\right\rangle \text { réel } \\
\left\langle U_{ \pm 1}^{(2)}\right\rangle=0
\end{array}\right.
$$

Pour que l'ellipsoïde soit de révolution, il faudrait en plus que, dans le système de ses axes principaux, $\left\langle U_{ \pm 2}^{(2)}\right\rangle$ soit nul.

Lorsque le spin $I$ a une valeur entière, l'ellipsoïde $\mathscr{E}_{\text {al }}$ peut, dans certains cas, subir une dégénérescence et devenir un cylindre de révolution (voir appendice II).

b) Section de l'ellipsoïde par un plan XOY quelconque contenant $O$. - Cette section est une ellipse. Considérons un point $Q$ quelconque de cette ellipse, défini par l'angle polaire $\varphi=(\mathrm{OX}, \mathrm{OQ})$, et appelons $I_{\varphi}$ la projection de $I$ sur OQ; on a :

$$
\begin{aligned}
\left\langle I_{\varphi}^{2}\right\rangle=\frac{1}{2}[ & \left\langle I_{X}^{2}+I_{Y}^{2}\right\rangle+\cos 2 \varphi\left\langle I_{X}^{2}-I_{Y}^{2}\right\rangle \\
& \left.+\sin 2 \varphi\left\langle I_{X} I_{Y}+I_{Y} I_{X}\right\rangle\right] . \quad \text { (II.D }
\end{aligned}
$$

Les axes de l'ellipse correspondent aux directions pour lesquelles $\left\langle I_{\varphi}^{2}\right\rangle$ est stationnaire, c'est-à-dire aux valeurs de $\varphi$ données par :

$$
\operatorname{tg} 2 \varphi=\frac{\left\langle I_{X} I_{Y}+I_{Y} I_{X}\right\rangle}{\left\langle I_{X}^{2}-I_{Y}^{2}\right\rangle}
$$

ou encore :

$$
\varphi=\frac{1}{2} \operatorname{Argt}\left\langle U_{2}^{(2)}\right\rangle+k \frac{\pi}{2}
$$

Soient $a$ et $b$ les longueurs du grand et du petit axe de l'ellipse; on a :

$$
\frac{1}{b^{2}}-\frac{1}{a^{2}}=\sqrt{\left\langle I_{X}^{2}-I_{Y}^{2}\right\rangle^{2}+\left\langle I_{X} I_{Y}+I_{Y} I_{X}\right\rangle^{2}}
$$

On peut construire géométriquement les deux axes de l'ellipse en utilisant une méthode classique [7] 
(voir fig. 3) : on sait qu'en général on peut faire passer par O deux plans $\mathrm{P}_{1}$ et $\mathrm{P}_{2}$ de section circulaire de $\mathscr{E}_{\text {al }}$, qui contiennent tous deux l'axe moyen de l'ellipsoïde; soient $\mathrm{D}_{1}$ et $\mathrm{D}_{2}$ les droites d'intersection de $\mathrm{P}_{1}$ et $\mathrm{P}_{2}$ avec le plan XOY : les axes de l'ellipse sont les bissectrices de $D_{1}$ et $D_{2}$. c) Propriétés diverses de l'ellipsö̈de. - Lorsque l'on donne des valeurs particulières aux quantités $\left\langle U_{q}^{(k)}\right\rangle$, l'ellipsoïde peut présenter diverses propriétés géométriques simples. Un certain nombre de cas particuliers, qui nous seront utiles dans la suite, sont rassemblés sur le tableau IV.

\section{TABLEAU IV}

1

$$
\begin{aligned}
& \left\langle U_{ \pm 2}^{(2)}\right\rangle=0 \\
& \left\langle U_{ \pm 1}^{(2)}\right\rangle=0 \\
& \left\langle U_{0}^{(2)}\right\rangle=0
\end{aligned}
$$

2

$\left\langle U_{ \pm 2}^{(2)}\right\rangle$ réel

$\left\langle U_{ \pm 1}^{(2)}\right\rangle=0$

3

$$
\left\langle U_{ \pm 2}^{(2)}\right\rangle=0
$$$$
\left\langle U_{ \pm 1}^{(2)}\right\rangle=0
$$

4

$$
\begin{array}{ll}
\left\langle U_{ \pm 1}^{(2)}\right\rangle=0 & \left\langle I_{x} I_{z}+I_{z} I_{x}\right\rangle=\left\langle I_{y} I_{z}+I_{z} I_{y}\right\rangle=0 \\
\left\langle U_{0}^{(2)}\right\rangle=0 & \left\langle I_{z}^{2}\right\rangle=\frac{1}{3} I(I+1)
\end{array}
$$

5

$$
\begin{array}{ll}
\left\langle U_{ \pm 2}^{(2)}\right\rangle=0 & \left\langle I_{x}^{2}\right\rangle=\left\langle I_{y}^{2}\right\rangle=\left\langle I_{z}^{2}\right\rangle=\frac{1}{3} I(I+1) \\
\left\langle U_{0}^{(2)}\right\rangle=0 & \left\langle I_{x} I_{y}+I_{y} I_{x}\right\rangle=0
\end{array}
$$

6

$$
\left\langle U_{ \pm 2}^{(2)}\right\rangle=0
$$$$
\left\langle I_{x}^{2}\right\rangle=\left\langle I_{y}^{2}\right\rangle
$$$$
\left\langle I_{x} I_{y}+I_{y} I_{x}\right\rangle=0
$$

7

$$
\left\langle U_{ \pm 1}^{(2)}\right\rangle=0
$$$$
\left\langle I_{x} I_{z}+I_{z} I_{x}\right\rangle=\left\langle I_{y} I_{z}+I_{z} I_{y}\right\rangle=0
$$

8

$$
\left\langle U_{ \pm 2}^{(2)}\right\rangle \text { réel } \quad\left\langle I_{x} I_{y}+I_{y} I_{x}\right\rangle=0
$$

9

$\left\langle U_{ \pm 2}^{(2)}\right\rangle$ imagi- $\quad\left\langle I_{x}^{2}\right\rangle=\left\langle I_{y}^{2}\right\rangle$

naire pur

$$
\left\langle I_{z} I_{x}+I_{x} I_{z}\right\rangle=0
$$

naire pur
$\mathscr{E}_{\text {al }}$ est la sphère de centre $\mathrm{O}$ et de rayon $\sqrt{3 / I(I+1)}$.

Ox, Oy, Oz sont les axes de $\mathscr{E}_{\mathrm{al}}$.

Ox, Oy, Oz sont les axes de $\mathscr{E}_{\text {al }}$, qui est de révolution autour de $\mathrm{Oz}$.

Oz est l'axe moyen de $\mathscr{E}_{\text {al }}$, de longueur $\sqrt{3 / I(I+1)} ; \mathrm{P}_{1}$ et $\mathrm{P}_{2}$ sont perpendiculaires.

$\mathrm{xOy}$ est le plan $\mathrm{P}_{1}$ de section circulaire (rayon du cercle : $\sqrt{3 / I}(\overline{I+1)})$; $\mathrm{P}_{2}$ contient $\mathrm{Oz}$.

$\mathrm{xOy}$ est le plan $\mathrm{P}_{1}$ de section circulaire.

Oz est un axe de $\mathscr{E}_{\text {al }}$.

Ox et Oy sont les axes de l'ellipse section de $\mathscr{E}_{\text {al }}$ par xOy.

Ox et Oy sont les bissectrices des axes de cette ellipse.

Oy et $\mathrm{Oz}$ sont les axes de l'ellipse section de $\mathscr{E}_{\text {al }}$ par yOz.

$\mathrm{Ox}$ et $\mathrm{Oz}$ sont les axes de l'ellipse section de $\mathscr{E}_{\text {al }}$ par xOz. 


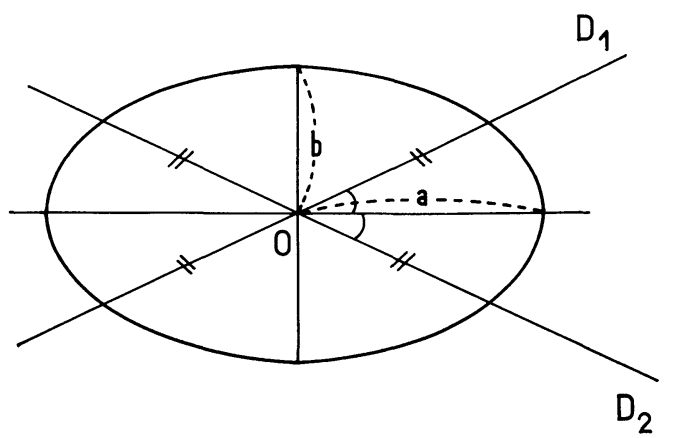

FIG. 3.

3. LuMiÈRE ABSORBÉE $L_{\mathrm{A}}\left(\mathbf{e}_{\lambda_{0}}\right)$ POUR UNE POLARISATION $\mathbf{e}_{\lambda_{0}}$ LINÉAIRE $\left({ }^{3}\right)$. - Lorsque le faisceau détecteur a une polarisation $\mathbf{e}_{\lambda_{0}}$ linéaire, on peut connaître la lumière absorbée $L_{A}\left(\mathbf{e}_{\lambda_{0}}\right)$ en utilisant uniquement l'ellipsoïde $\mathscr{E}_{\text {al }}$. En effet, $L_{\mathrm{A}}\left(\mathbf{e}_{\lambda_{0}}\right)$ comprend trois parties : la première qui correspond à la population totale de l'état fondamental, qui ne dépend ni de $\boldsymbol{e}_{\lambda_{\mathrm{p}}}$, ni de $\sigma_{\mathrm{f}}$, et qui est donc une constante (cf. $\S$ II.B); la seconde qui correspond à l'orientation, qui est nulle (cf. § II. C) ; enfin, la dernière qui dépend de l'alignement, que nous allons étudier, et qui est proportionnelle à :

$$
\left\langle\mathbf{e}_{\lambda_{0}}\left|B^{(2)}\right| \mathbf{e}_{\lambda_{0}}\right\rangle=\left\langle\mathbf{e}_{\lambda_{0}}\left|M^{(2)}\right| \mathbf{e}_{\lambda_{0}}\right\rangle
$$

avec, d'après (II.D.1) :

$$
\begin{aligned}
& B^{(2)}=P_{X Y} M^{(2)} P_{X Y} \\
& =c^{(2)}\left\{\begin{array}{cc}
\left\langle I_{X}^{2}\right\rangle & (1 / 2)\left\langle I_{X} I_{Y}+I_{Y} I_{X}\right\rangle \\
(1 / 2)\left\langle I_{X} I_{Y}+I_{Y} I_{X}\right\rangle & \left\langle I_{Y}^{2}\right\rangle
\end{array}\right) \\
& -(1 / 3) I(I+1)\} \text {. }
\end{aligned}
$$

Si nous choisissons comme axe OX la direction de la polarisation incidente $\left|\mathbf{e}_{\lambda_{0}}\right\rangle$, nous avons donc : $\left\langle\mathbf{e}_{\lambda_{0}}\left|B^{(2)}\right| \mathbf{e}_{\lambda_{0}}\right\rangle=c^{(2)}\left[\left\langle I_{X}^{2}\right\rangle-(1 / 3) I(I+1)\right] \quad$ (II.D.17) $L_{\mathrm{A}}\left(\mathbf{e}_{\lambda_{0}}\right)$ ne dépend donc que de l'alignement dans la direction de la polarisation lumineuse. L'ellipsoïde des alignements permet ainsi de voir très rapidement comment varie la lumière absorbée pour une polarisation plane quelconque. Par exemple, le grand axe et le petit axe correspondent aux polarisations qui sont le plus et le moins absorbées. Le dichroïsme linéaire de la vapeur est donc maximum pour la direction de propagation du faisceau lumineux qui est parallèle à l'axe moyen de $\mathscr{E}_{\text {al }}$.

4. Polarisations principales, dichroÏsme et biréFRINGENGE D'UNE VAPEUR, DANS LE GAS OU L'ORIENTATION EST NULLE. - Considérons une direction de

(3) On montre dans JP1 et JP2 [8] qu'il existe une grande symétrie entre l'expression de $L_{\mathrm{A}}\left(\mathbf{e}_{\lambda_{0}}\right)$, lumière absorbée par la vapeur, et celle de $L_{\mathrm{F}}\left(\mathbf{e}_{\lambda}\right)$, lumière de fluorescence émise par la vapeur. Cette symétrie permet de transposer immédiatement la plupart des résultats de cette partie à l'étude de la polarisation de la lumière de fluorescence émise par des atomes alignés dans l'état excité. propagation $\mathrm{OZ}$ quelconque. Les polarisations principales de la vapeur correspondent aux vecteurs propres de l'opérateur $B=P_{X Y} M P_{X Y}$. Lorsque l'orientation est nulle, la matrice $M^{(1)}$ l'est aussi; $M^{(0)}$ étant proportionnelle à la matrice unité, les polarisations principales sont donc simplement les vecteurs propres de l'opérateur $B^{(2)}=P_{X Y} M^{(2)} P_{X Y}$. On voit sur (II.D.16) que $B^{(2)}$ est une matrice symétrique réelle; les polarisations propres sont donc des polarisations linéaires $|\mathbf{a}\rangle$ et $|\mathbf{b}\rangle$ orthogonales entre elles.

$\mathrm{Si}$ nous choisissons comme axes $\mathrm{OX}$ et $\mathrm{OY}$ les directions de $|\mathbf{a}\rangle$ et $|\mathbf{b}\rangle$, nous obtenons, en écrivant que la matrice $B^{(2)}$ donnée en (II.D.16) est diagonale:

$$
\left\langle I_{X} I_{Y}+I_{Y} I_{X}\right\rangle=0 .
$$

On voit donc (cf. égalité (II.D.13)) que $|\mathbf{a}\rangle$ et $|\mathbf{b}\rangle$ sont portés par les axes de l'ellipse intersection de l'ellipsoïde des alignements avec le plan XOY.

Le dichroïsme et la biréfringence de la vapeur, c'est-à-dire la différence $n_{a}-n_{b}$ entre les deux indices principaux, sont proportionnels à la différence des alignements dans les deux directions $|\mathbf{a}\rangle$ et $|\mathbf{b}\rangle$; si $a$ et $b$ sont les longueurs des deux axes de l'ellipse, liées par la relation (II.D.15), on a :

$$
n_{a}-n_{b} \propto \frac{1}{b^{2}}-\frac{1}{a^{2}} \text {. }
$$

La règle pour construire les polarisations principales d'une vapeur alignée est donc simple : on considère l'intersection de l'ellipsoïde des alignements avec le plan passant par $\mathrm{O}$, perpendiculaire à la direction de propagation du faisceau lumineux; les polarisations principales sont portées par les axes de l'ellipse ainsi obtenue; la différence relative des deux indices principaux augmente avec l'excentricité de l'ellipse. Pour déterminer géométriquement les polarisations principales, on peut utiliser la construction rappelée au $\S$ D.2.b.

Si le plan XOY est confondu avec un des deux plans de section circulaire $\mathrm{P}_{1}$ ou $\mathrm{P}_{2}$, c'est-à-dire si $\left\langle\left(I_{X}+i I_{Y}\right)^{2}\right\rangle=0$ (cf. $\S \mathrm{D} .2$.c), la vapeur se comporte optiquement comme un milieu isotrope. Il existe donc deux directions de propagation $\mathrm{OZ}_{1}$ et $\mathrm{OZ}_{2}$ pour lesquelles la vapeur alignée ne présente ni dichroïsme, ni biréfringence; $\mathrm{OZ}_{1}$ et $\mathrm{OZ}_{2}$ sont contenues dans le plan du grand axe et du petit axe de $\mathscr{E}_{\text {al }}$. S'il existe un axe autour duquel $\mathscr{E}_{\text {al }}$ est de révolution, $\mathrm{OZ}_{1}$ et $\mathrm{OZ}_{2}$ sont confondues avec cet axe.

5. Application aux Gas Particuliers ÉtudiÉs dans LA PREMIÈre PARTIE. - Nous allons revenir sur les deux cas particuliers étudiés au § I. G, et montrer que les résultats obtenus alors pour les signaux correspondant à l'alignement peuvent être retrouvés géométriquement, en utilisant l'ellipsoïde $\mathscr{E}_{\text {al }}$. Nous considérons donc une vapeur soumise au pompage optique dans les conditions qui ont été définies en I.G. On sait qu'alors la variation dans le temps de la matrice 
densité $\sigma_{\mathrm{f}}(t)$ correspond à une rotation uniforme de vitesse angulaire $\omega$ autour de $\mathbf{H}_{0}$. L'évolution de l'ellipsoïde $\mathscr{E}_{\text {al }}$ est donc très simple : il tourne sans se déformer, avec une vitesse constante, autour de $\mathbf{H}_{\mathbf{0}}$.

a) Lorsque le faisceau lumineux se propage parallèlement au champ magnétique $\mathbf{H}_{0}$, l'axe de rotation est parallèle à la direction du faisceau lumineux, de sorte que l'intersection de $\mathscr{E}_{\text {al }}$ avec le plan XOY est une ellipse qui tourne, sans se déformer, à la pulsation $\omega$ autour de son centre $O$. Les polarisations principales se déplacent de la même manière, et la vapeur est équivalente à une lame biréfringente et dichroïque, dont les indices principaux sont constants, et qui tourne autour de $\mathbf{H}_{\mathbf{0}}$ à la vitesse angulaire $\omega$.

Il est clair que, lorsque cette lame a tourné de $180^{\circ}$, elle se trouve dans une position équivalente, du point de vue optique, à sa position de départ : les signaux modulés ont donc la pulsation $2 \omega$. De plus, on voit qu'une modification de l'angle $\Psi_{1}$ qui définit la polarisation plane incidente revient à un simple changement de l'origine des temps : les signaux dépendent donc de $t$ et $\Psi_{1}$ par l'intermédiaire de la quantité $2\left(\omega t-\Psi_{1}\right)$. Enfin, on remarque que les signaux dus aux transitions réelles sont maximaux (ou minimaux) au moment où les axes de la lame biréfringente sont parallèles à la direction $\Psi_{1}$; au contraire, ceux qui sont dus aux transitions virtuelles, obtenus en mesurant le degré de polarisation circulaire du faisceau lumineux transmis, sont maximals (ou minimals) au moment où les axes de la lame font un angle de $45^{\circ}$ avec la direction $\Psi_{1}$. L'ensemble de ces remarques permet de conclure qu'avec un faisceau résonnant le signal modulé est proportionnel à $\cos \left(2 \omega t-2 \Psi_{1}\right)$, et avec un faisceau non résonnant à $\sin \left(2 \omega t-2 \Psi_{1}\right)$; nous retrouvons donc bien tous les résultats du $\S \mathrm{I}$. C. 1 de l'article précédent [1].

b) Lorsque le faisceau lumineux se propage perpendiculairement au champ magnétique $\mathbf{H}_{0}$, l'interprétation géométrique est en général moins simple que dans le cas précédent. Les plans $\mathrm{P}_{1}$ et $\mathrm{P}_{2}$ tournent autour de l'axe $\mathrm{Oz}$, contenu dans le plan XOY (cf. fig. 2), de sorte que les droites $\mathrm{D}_{1}$ et $\mathrm{D}_{2}$ effectuent un mouvement oscillant compliqué dans ce plan. Les axes de l'ellipse, ainsi que sa forme, varient donc au cours du temps. Nous allons voir toutefois que, lorsque l'on suppose que seules certaines composantes de l'alignement sont non nulles, on peut obtenir géométriquement quelques résultats simples.

Modulations lumineuses à la pulsation $2 \omega$. - Ces modulations sont dues aux oscillations des valeurs moyennes $\left\langle U^{(2)}\right\rangle$; il est donc possible de les étudier en supposant que les valeurs moyennes $\left\langle U_{ \pm 1}^{(2)}\right\rangle$ sont nulles $\left({ }^{4}\right)$. Dans ce cas (cf. tableau IV, propriété 7), Oz est un des trois

(4) Rappelons que dans tout cet article nous supposons que l'épaisseur optique de la vapeur est faible ; les signaux optiques dépendent donc linéairement des grandeurs $\left\langle U_{q}^{(k)}\right\rangle$. axes de $\mathscr{E}_{\text {al }}$; les axes de l'ellipse sont alors OX et OY (voir fig. 4). La longueur $a$ de l'axe porté par OX est constante, alors que la longueur $b$ de l'axe porté par OY varie périodiquement dans le temps avec la

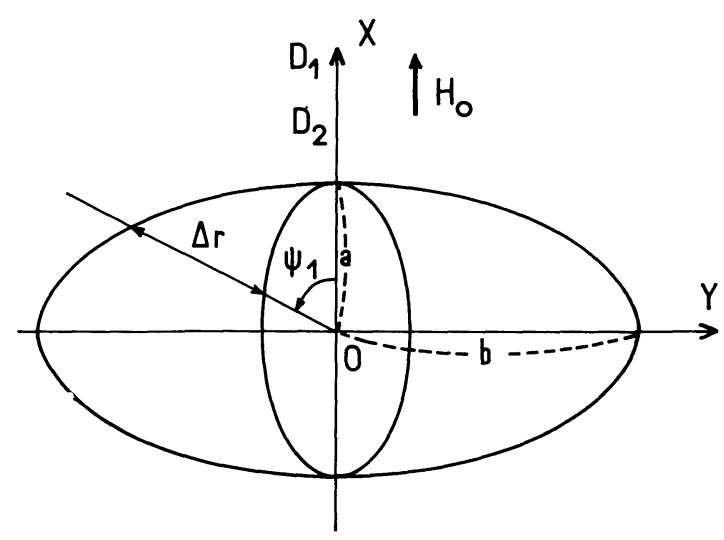

FIG. 4.

pulsation $2 \omega$ (en effet, $\mathscr{E}_{\text {al }}$ est invariant dans une rotation de $180^{\circ}$ autour de $\mathrm{Oz}$ ). Nous sommes donc dans un cas complémentaire de celui qui vient d'être étudié : les polarisations principales de la vapeur sont fixes (parallèle et perpendiculaire à $\mathbf{H}_{0}$ ) et c'est la différence des deux indices principaux qui évolue à la pulsation $2 \omega$ (l'un des deux indices restant constant)

Avec un faisceau résonnant polarisé linéairement, l'amplitude de la modulation à $2 \omega$ observée est proportionnelle à la variation de l'alignement dans la direction $\Psi_{1}$, qui est liée à la variation $\Delta r$ du rayon vecteur correspondant de l'ellipse (cf. fig. 4). Or l'équation de cette ellipse est :

$$
\frac{1}{r^{2}}=\frac{\cos ^{2} \Psi_{1}}{a^{2}}+\frac{\sin ^{2} \Psi_{1}}{b^{2}} .
$$

La variation de l'alignement est proportionnelle à : $\Delta\left(1 / r^{2}\right)=\sin ^{2} \Psi_{1} \Delta\left(1 / b^{2}\right)$. L'amplitude de la modulation à $2 \omega$ varie donc en fonction de $\Psi_{1}$ comme $\sin ^{2} \Psi_{1}$.

Avec un faisceau non résonnant polarisé linéairement et un analyseur circulaire, on détecte un signal proportionnel aux taux de polarisation circulaire de la lumière transmise. On voit que, lorsque $\Psi_{1}^{\circ}=0$ ou $\pi / 2$, ce taux est nul; on montre aisément que, lorsque $\Psi_{1}$ est quelconque, il est proportionnel à $\sin 2 \Psi_{1}$.

Nous retrouvons donc tous les résultats du $\S$ I.C.2.a de la première partie de cette étude [1].

Modulations lumineuses à la pulsation $\omega$. - Pour étudier ces modulations, nous allons supposer que les seules composantes de l'alignement non nulles sont les composantes $\left\langle U_{ \pm 1}^{(2)}\right\rangle$. Dans ce cas (cf. tableau IV, propriété 5 ), l'un des plans de section circulaire de $\mathscr{E}_{\mathrm{al}}$ est $\mathrm{xOy}$, tandis que l'autre contient $\mathrm{Oz}$. Les droites $\mathrm{D}_{1}$ et $\mathrm{D}_{2}$ sont donc confondues avec $\mathrm{OX}$ et $\mathrm{OY}$, de sorte que les axes de l'ellipse qui nous intéresse sont à $45^{\circ}$ 


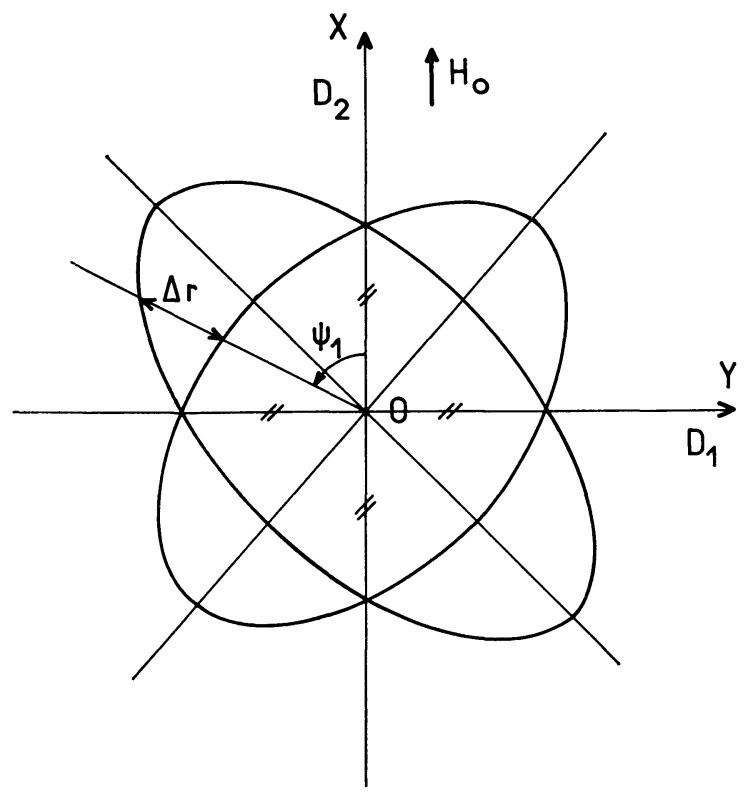

FIG. 5.

de OX et OY (voir fig. 5). Les polarisations principales sont donc dans ce cas à $45^{\circ}$ du champ magnétique $\mathbf{H}_{0}\left({ }^{5}\right)$.

Les quatre points de l'ellipse sur les axes OX et OY sont fixes; on montre aisément que ceci entraîne que les longueurs $a$ et $b$ des deux axes de l'ellipse sont reliées par la relation $1 / a^{2}+1 / b^{2}=$ constante. La somme des deux indices principaux est constante; c'est leur différence qui est modulée à la pulsation $\omega$.

Comme plus haut, on calcule la variation de l'alignement dans la direction $\Psi_{1}$ en utilisant l'équation de l'ellipse :

$$
1 / r^{2}=\frac{1}{2}\left[1 / a^{2}+1 / b^{2}\right]+\frac{1}{2} \sin 2 \Psi_{1}\left[1 / a^{2}-1 / b^{2}\right] .
$$

On a donc :

$$
\Delta\left(1 / r^{2}\right)=\frac{1}{2} \sin 2 \Psi_{1} \Delta\left(1 / a^{2}-1 / b^{2}\right)
$$

et l'amplitude des signaux modulés à la pulsation $\omega$, obtenus avec un faisceau résonnant polarisé linéairement, est proportionnelle à $\sin 2 \Psi_{1}$.

On peut également étudier le cas où le faisceau détecteur est non résonnant et polarisé linéairement. On constate alors que la disposition des axes de la lame biréfringente équivalente est telle que les modulations lumineuses de pulsation $\omega$ sont proportionnelles à $\cos 2 \Psi_{1}$.

Nous retrouvons donc bien tous les résultats du $\S$ I.G.2.b de la première partie [1].

E. Cas général. - Dans le cas général, la vapeur possède à la fois de l'orientation et de l'alignement

(5) Nous retrouvons ici un résultat démontré par ailleurs par Pancharatnam [9]. dans l'état fondamental. Nous nous proposons dans ce paragraphe de montrer comment on peut alors construire les polarisations principales de la vapeur, pour une direction de propagation $\mathrm{OZ}$ quelconque. Nous choisissons comme axes OX et OY les axes de l'ellipse intersection de $\mathscr{E}_{\text {al }}$ par le plan perpendiculaire à $\mathrm{OZ}$, parallèles à $|\mathbf{a}\rangle$ et $|\mathbf{b}\rangle$, de façon à avoir (cf. II.D.13) :

$$
\left\langle I_{X} I_{Y}+I_{Y} I_{X}\right\rangle=0 \text {. }
$$

En reportant (II.E.1) dans l'équation (III.14) de JP4 qui permet de calculer les polarisations principales sous la forme $\mathrm{e}^{-i \theta} \cos \varphi\left|\mathbf{e}_{X}\right\rangle+\mathrm{e}^{i \theta} \sin \varphi\left|\mathbf{e}_{Y}\right\rangle$, on obtient :

$$
\begin{aligned}
-c^{(\mathbf{2})} \sin 2 \varphi & \left\langle I_{X}^{2}-I_{Y}^{2}\right\rangle \\
& +\sqrt{2} c^{(\mathbf{1})}[\cos 2 \varphi \sin 2 \theta-i \cos 2 \theta]\left\langle I_{Z}\right\rangle=0
\end{aligned}
$$

ou encore :

$$
\left\{\begin{aligned}
\theta & =\pi / 4 \\
\operatorname{tg} 2 \varphi & =\frac{c^{(1)} \sqrt{2}}{c^{(2)}} \frac{\left\langle I_{Z}\right\rangle}{\left\langle I_{X}^{2}-I_{Y}^{2}\right\rangle} .
\end{aligned}\right.
$$

Ces résultats montrent que les deux polarisations principales de la vapeur sont en général elliptiques. Les axes des deux ellipses correspondantes sont parallèles aux directions de $|\mathbf{a}\rangle$ et $|\mathbf{b}\rangle$, obtenues à partir de $\mathscr{E}_{\text {al }}$ seul; le rapport entre les longueurs des axes est égal à tg $\varphi$, qui dépend à la fois de l'orientation et de l'alignement.

La différence entre les indices principaux se calcule à l'aide de l'égalité (III.13) de JP4; on obtient : $n_{1}-n_{2}$

$\propto \sqrt{\left[c^{(2)}\left\langle I_{X}^{2}-I_{Y}^{2}\right\rangle\right]^{2}+2\left[c^{(1)}\left\langle I_{Z}\right\rangle\right]^{2}}$.

Les relations qui viennent d'être écrites peuvent s'interpréter physiquement de la manière suivante : considérons un faisceau lumineux qui traverse successivement un polariseur linéaire faisant avec la direction du vecteur $|\mathbf{a}\rangle$ un angle $\varphi$ défini à $\pi / 2$ près par la seconde des relations (II.E.3), puis une lame quart d'onde, d'axes parallèles à $|\mathbf{a}\rangle$ et $|\mathbf{b}\rangle$ (axe rapide $|\mathbf{a}\rangle)$; la polarisation du faisceau ainsi obtenue est une des polarisations principales de la vapeur, de sorte qu'elle n'est pas modifiée lors de la traversée de la cellule.

Lorsque $\left\langle I_{Z}\right\rangle=0$, les relations (II.E.3) donnent $\varphi=0, \pi / 2$; nous retrouvons bien comme polarisations principales deux polarisations planes parallèles aux vecteurs $|\mathbf{a}\rangle$ et $|\mathbf{b}\rangle$. Pour toutes les directions de propagation perpendiculaires à $\langle I\rangle$, les polarisations principales sont planes.

Lorsque $\left\langle I_{X}^{2}\right\rangle=\left\langle I_{Y}^{2}\right\rangle$, on a $\varphi=\pi / 4,3 \pi / 4$; les polariseurs que nous avons décrits correspondent aux polarisations circulaires. Il existe en général deux directions de propagation pour lesquelles la vapeur présente un dichroïsme et une biréfringence circulaires : ce sont les directions $\mathrm{OZ}_{1}$ et $\mathrm{OZ}_{2}$ définies au $§$ II.D.4. 
La relation (II.E.4) montre qu'en général il n'existe pas de direction de propagation pour laquelle la vapeur ne présente aucune anisotropie optique. Ce cas ne peut se produire que si l'on a, à la fois :

$$
\left\{\begin{array}{l}
\left\langle I_{Z}\right\rangle=0 \\
\left\langle I_{X}^{2}\right\rangle=\left\langle I_{Y}^{2}\right\rangle
\end{array}\right.
$$

c'est-à-dire si l'orientation dans l'état fondamental est soit nulle, soit contenue dans l'un des deux plans $\mathrm{P}_{\mathbf{1}}$ ou $\mathrm{P}_{2}$ de section circulaire de l'ellipsoïde des alignements.

Conclusion. - Nous avons étudié de manière pratique et détaillée les divers signaux que l'on peut obtenir avec un faisceau lumineux auxiliaire dans une expérience de pompage optique, en nous limitant au cas de l'effet paramagnétique. Cette étude a mis en évidence la manière dont on peut isoler dans le signal optique ainsi obtenu l'influence de chaque observable de l'état fondamental, au moyen d'un analyseur et d'un polariseur convenables. Nous avons montré que l'ensemble des signaux, qui a priori dépendent de manière complexe des $(2 I+1)^{2}$ éléments de la matrice densité $\sigma_{\mathrm{f}}$, peut être ramené, quelle que soit la valeur de $I$, à un nombre relativement restreint de signaux correspondant soit à l'alignement, soit à l'orientation, et dont les variations en fonction de la nature du polariseur et de l'analyseur sont très simples. Deux méthodes ont été utilisées dans ce but : la première consiste en un calcul qui est basé sur le formalisme des opérateurs tensoriels irréductibles; la deuxième, plus concrète, fait intervenir diverses représentations géométriques, et permet d'aboutir aux résultats pratiquement sans calculs. Cette dernière méthode devrait pouvoir être appliquée avec succès à l'étude d'autres problèmes que ceux qui ont été abordés dans cet article.

Remerciements. - Les auteurs tiennent à remercier vivement M. C. Cohen-Tannoudji pour son aide et ses conseils qui ont été très utiles à l'élaboration de ces articles. Ils remercient également le rapporteur de la Commission des Publications du Journal de Physique pour les diverses remarques utiles faites lors de la lecture du manuscrit.

\section{APPENDICE II}

Dégénérescences de l'ellipsoïde $\mathscr{E}_{\mathrm{al}}$. - Soient $\mid \mu(\Omega)>$ les vecteurs propres de $I_{\Omega}$. Les éléments de la matrice densité $\sigma_{\mathrm{f}}$ s'écrivent, dans la base correspondante :

$$
\sigma_{\mu \mu^{\prime}}(\Omega)=\left\langle\mu(\Omega)\left|\sigma_{\mathrm{f}}\right| \mu^{\prime}(\Omega)\right\rangle .
$$

$\sigma_{\mathrm{f}}$ est un opérateur hermitique, défini positif; on montre aisément que ces propriétés entraînent l'inégalité :

$$
\left|\sigma_{\mu \mu^{\prime}}(\Omega)\right|^{2} \leqslant \sigma_{\mu \mu}(\Omega) \cdot \sigma_{\mu^{\prime} \mu^{\prime}}(\Omega) .
$$

L'alignement dans la direction $\Omega$ est donné par :

$$
\left\langle I_{\Omega}^{2}\right\rangle=\sum_{\mu=-I}^{+I} \mu^{2} \sigma_{\mu \mu}(\Omega)
$$

avec :

$$
|\mu| \leqslant I ; \quad \sum_{\mu} \sigma_{\mu \mu}(\Omega)=1 .
$$

On a donc :

$$
0 \leqslant\left\langle I_{\Omega}^{2}\right\rangle \leqslant I^{2} .
$$

On voit sur (III.9) que $\left\langle I_{\Omega}^{2}\right\rangle$ ne peut être nul que lorsque $I$ a une valeur entière, et que toutes les populations $\sigma_{\mu \mu}(\Omega)$ sont nulles, sauf $\sigma_{00}(\Omega)$. L'inégalité (III.8) entraîne alors que toutes les cohérences $\sigma_{\mu \mu^{\prime}}(\Omega)\left(\mu \neq \mu^{\prime}\right)$ sont nulles.

En général, il n'existe pas de direction $\Omega_{0}$ pour laquelle $\left\langle I_{\Omega_{0}}^{2}\right\rangle$ soit nul. Si toutefois cette direction existe, elle correspond à un vecteur propre de $\mathscr{M}^{(2)}$ dont la valeur propre est nulle; l'ellipsoïde $\mathscr{E}_{\text {al }}$ est donc dégénéré en un cylindre ayant $\Omega_{0}$ pour axe.

La nullité des cohérences $\sigma_{\mu \mu^{\prime}}\left(\Omega_{0}\right)$ entraîne que $\sigma_{\mathrm{f}}$ est invariante par rotation autour de $\Omega_{0}$ : le cylindre est donc de révolution autour de son axe; son rayon est $\sqrt{2 / I(I+1)}$.

La dégénérescence de $\mathscr{E}_{\text {al }}$ ne peut être plus élevée que celle qui vient d'être décrite; en effet, si deux valeurs propres de $\mathscr{M}^{(2)}$ étaient nulles, la troisième aurait pour valeur $\operatorname{Tr}\left\{\mathscr{M}^{(2)}\right\}=I(I+1)>I^{2}$, ce qui est contradictoire avec la deuxième inégalité de (III.10).

\section{BIBLIOGRAPHIE}

[1] Lalö̈ (F.), Leduc (M.) et Minguzzi (P.), J. Physique, 1969, 30, 277.

[2] Dehmelt (H. G.), Phys. Rev., 1957, 105, 1924.

[3] Bfil. (W. E.) et Bloom (A. L.), Phys. Rev., 1957, 107, 1559.

[4] Manuel (J.) et Cohen-Tannoudji (C.), C. R. Acad. Sci., 1963, 257, 413.

Manuel (J.), Diplôme d'études supérieures, Paris, 1964.

[5] Cohen-Tannoudji (C.) et Laloë (F.), J. Physique, $1967,28,505$ et 722 . Ces deux articles sont désignés dans le texte par JP3 et JP4.
[6] Happer (W.) et Mathur (B. S.), Phys. Rev., 1967, $163,12$.

Happer (W.), Compte rendu de 1' "International Conference on Optical Pumping and Atomic Line Shape " (O.P.A.L.S.), Varsovie (Pologne), 1968.

[7] Bruhat (G.) et Kastlek (A.), Optique, 5e édition, édit. Masson, chap. XX, p. 409.

[8] Barrat (J.-P.) et Cohen-Tannoudji (C.), J. Physique Rad., 1961, 22, 329 et 443. Ces deux articles sont désignés dans le texte par JP1 et JP2.

[9] Pancharatnam (S.), Phys. Lett., 1968, 27 A, 509, et Compte rendu de 1' "International Conference on Optical Pumping and Atomic Line Shape" (O.P.A.L.S.), Varsovie (Pologne), 1968. 